\title{
Wage-led ou profit-led? \\ Análise das estratégias de crescimento das economias sob o regime de metas de inflação, câmbio flexível, mobilidade de capitais e endividamento externo *
}

\author{
Flávio A. C. Basilio ** \\ José Luís da Costa Oreiro ${ }^{* * *}$
}

\begin{abstract}
Resumo
Neste artigo apresentamos um modelo macrodinâmico de inspiração pós-keynesiana com o objetivo de analisar os efeitos da abertura da conta de capitais sobre a performance macroeconômica das economias em desenvolvimento. Neste particular, os exercícios de estática comparativa mostram que um aumento do endividamento externo pode ter efeitos benéficos para a economia no curto prazo, uma vez que induz o aumento do grau de utilização da capacidade produtiva e da taxa de crescimento do estoque de capital. No entanto, a dinâmica de longo prazo mostra que, sob certas condições, o acesso ao mercado internacional de capitais pode gerar trajetórias explosivas para o sistema econômico, notadamente em um contexto em que a política monetária é conduzida com base em um regime de metas de inflação. Além disso, demonstra-se que uma redução da meta de inflação perseguida pelo Banco Central, dentro do contexto de abertura da conta de capitais e de captação externa por parte das empresas, pode gerar um aumento permanente da taxa real de juros, bem como um aumento do próprio custo do endividamento externo da economia contribuindo, assim, para uma maior fragilidade externa. Ademais, analisou-se a consistência das estratégias de crescimento das economias desenvolvidas e em desenvolvimento. No geral, os resultados encontrados mostram a validade da Lei de Thirlwall, de modo que o regime de crescimento das economias depende, em última análise, das restrições impostas pelas elasticidades-renda das importações e exportações.
\end{abstract}

Palavras-chave: Crescimento endógeno; Poupança externa; Câmbio flexível; Wage-led; Profit-led.

\section{Abstract \\ Wage-led or profit-led? An analysis of the growth strategies of economies under the regime of inflation targeting, a flexible exchange rate, capital mobility and external debt}

In this paper, a post-keynesian macrodynamic model is presented in order to analyze the effects of capital account openness on the macroeconomic performance of developing economies. In particular, the exercise of comparative statics shows that an increase in external indebtedness may have beneficial effects on the economy in the short-term, since it induces an increase in the degree of capacity utilization and the growth rate of capital stock. The long-run dynamics, however, show that, under certain conditions, access to the international capital market can generate explosive trajectories for the economic system, especially in a context where monetary policy is conducted in an inflation-targeting

\footnotetext{
* Trabalho recebido em 19 de julho de 2013 e aprovado em 8 de setembro de 2014.

** Professor do Centro Universitário UDF, Brasília, DF, Brasil. E-mail: flaviobasilio@ gmail.com.

**** Pesquisador 1B do CNPq (Conselho Nacional de Desenvolvimento Científico e Tecnológico), Rio de
} Janeiro, RJ, Brasil. E-mail: jlcoreiro@terra.com.br. Os autores agradecem os comentários e sugestões dos pareceristas. 
regime. In addition, it shows that a reduction in the inflation target pursued by the Central Bank - in the context of capital account opening and foreign funding on the part of enterprises - can generate a permanent increase in the real rate of interest. An increase in the enterprise's own cost as a result of the economy's external indebtedness, contributing to greater external fragility, also occurs. Furthermore, we analyzed the consistency of the growth strategies of developed and developing economies. Overall, the results show the validity of Thirlwall's Law, in that the growth regime of economies ultimately depends on the restrictions imposed by the income elasticities of imports and exports.

Keywords: Endogenous growth; External saving; Exchange rate regime; Wage-led; Profit-led. JEL F43, O11, F41, E12

\section{Introdução}

Os países em desenvolvimento têm procurado adotar políticas que acelerem o crescimento econômico, de forma a aumentar o bem-estar de seus cidadãos e reduzir a pobreza. Dentro deste contexto, diversas teorias e modelos têm sido formulados com o intuito de identificar os fatores de crescimento das economias capitalistas. No âmbito das teorias do crescimento econômico de inspiração neoclássica, desenvolvidas a partir do trabalho seminal de Solow (1956), a poupança é vista como um elemento essencial para o crescimento de longo prazo.

Nesse contexto, os países deveriam recorrer à poupança externa para aumentar a sua taxa de poupança e, dessa forma, acelerar o seu crescimento de longo prazo. Esta interpretação, explicita ou implicitamente, também é apoiada do ponto de vista teórico pelo modelo de liberalização financeira de E. Shaw (1973) e R. McKinnon (1973). Os pilares do modelo são as relações de causalidade entre a taxa de poupança da economia e sua taxa de crescimento, segundo a qual de taxas de juros mais altas aumentam as taxas de poupança criando, desse modo, mais recursos disponíveis para investimento e crédito. Seguindo esta linha teórica, pelo fato de existirem taxas de juro baixas, determinadas institucionalmente e não por meio dos mecanismos de mercado, dá-se origem a uma situação conhecida por "repressão financeira", em que a taxa de juros de equilíbrio está abaixo do ponto em que se deveria encontrar. Como consequência, o investimento é baixo e o crescimento econômico ressentir-se-á disso.

O pressuposto teórico dessa análise é que, na ausência de restrições à mobilidade internacional de capital, os capitais se moverão dos países ricos para os países pobres ${ }^{1}$, ajudando assim a financiar um volume maior de investimento nos países em desenvolvimento, o que contribuiria decisivamente para a aceleração do crescimento. Esse processo decorre do fato de que os capitais internacionais tendem a fluir para os países que ofereçam maior remuneração (cf. Lucas, 1990). Sendo a remuneração do capital maior nos países em desenvolvimento, onde ele é escasso,

(1) Eliminadas as restrições à mobilidade de capitais, haveria um aumento contínuo nas transações cambiais e nos fluxos de capitais internacionais, beneficiando os países em desenvolvimento. 
do que nos países desenvolvidos, onde ele é abundante, os capitais se deslocariam naturalmente dos países ricos para os países pobres, contribuindo para a atenuação das desigualdades econômicas internacionais. Esta visão pró-liberalização da conta de capitais se fundamenta, portanto, na hipótese de que os capitais externos são considerados endógenos ao processo de crescimento econômico, uma vez que fluxos livres de capital promovem a eficiente alocação internacional dos recursos, com capitais fluindo em larga escala de países com elevada relação capital/trabalho para países com baixa relação capital/trabalho.

A ideia de que a poupança externa é condição necessária para o desenvolvimento econômico, no entanto, não é consenso entre os economistas. Em oposição a estes argumentos, destacam-se os trabalhos de Rodrik (1998) e Bhagwati (1998). Segundo estes autores, não há qualquer evidência de que países que se financiaram por intermédio de capitais externos melhoraram a performance de crescimento do produto e/ou tenham obtido melhorias no perfil de distribuição de renda. Uma segunda linha de argumentação, contra a tese de crescimento com poupança externa, foi elaborada por Bresser e Nakano (2003) e Oreiro (2004). Para esses autores, o financiamento via poupança externa tende a gerar uma redução da taxa de crescimento de longo prazo das economias dos países em desenvolvimento devido ao aumento explosivo do endividamento externo decorrente dessa estratégia de crescimento.

Com efeito, um importante aspecto do debate sobre estratégias de crescimento econômico diz respeito aos efeitos que o câmbio e o endividamento externo possuem sobre a distribuição funcional da renda, principalmente devido aos aspectos intimamente relacionados entre câmbio real e participação dos salários na renda. Sobre esse tema, a literatura convencional falha em sua análise por desconsiderar tais efeitos e relações.

Os salários possuem dupla função em qualquer economia capitalista. De um lado, constituem custos para as empresas, como argumentado pela literatura convencional. De outro, são um importante componente da demanda agregada. De acordo com a tradição Kaleckiana, com expoente nos trabalhos de Kalecki (1971), Steindl (1952) e Bhaduri e Marglin (1990), os salários atuam nas duas pontas, atuando como custos e também como fonte de demanda. Contudo, existiria uma relação inequívoca entre a expansão da participação dos trabalhadores na renda e os efeitos expansionistas sobre a economia, prevalecendo a tradição de defesa do regime wage-led. Ademais, a distinção entre os regimes wage-led e profit-led é um tema central do referencial pós-keynesiano (Bhaduri e Marglin 1990).

Sob a perspectiva de economias abertas, tem-se, ainda, que levar em consideração os efeitos da distribuição funcional da renda entre capitalistas e trabalhadores, não apenas sobre consumo e investimento, como demonstrado por 
Rowthorn (1981), Taylor (1983) e Dutt (1987), mas também sobre as transações correntes. Assim, para saber se uma economia é wage-led ou profit-led no total, temse que avaliar os efeitos de mudanças na distribuição funcional da renda sobre as transações correntes, como tem sido sublinhado por Blecker $(1989,2011)$, bem como por Bhaduri e Marglin (1990).

Em geral, uma economia fechada que é profit-led, também é profit-led (totalmente profit-led) quando sujeita à abertura das transações com o resto do mundo. Contudo, uma economia fechada wage-led, não necessariamente será totalmente wage-led quando sujeita à competição internacional. Isto porque, para economias abertas, o efeito do aumento da participação dos salários na renda sobre as transações correntes depende da estrutura da elasticidade-renda das importações e das exportações. Com efeito, se a elevação dos salários não é acompanhada da elevação do preço dos produtos exportados, este resultado levará à redução das margens de lucro dos capitalistas, com eventual perda de competitividade das exportações, antes rentáveis. Este é essencialmente o caso de profit squeeze, em que as margens de lucro são comprimidas entre custos domésticos, de um lado, e da concorrência externa, do outro.

Verifica-se, portanto, que a abertura da economia gera complexidade na avaliação das mudanças na distribuição funcional da renda. O impacto favorável no mercado interno de um aumento na parcela salarial pode ser revertido ao se considerar os efeitos sobre as transações correntes.

Neste sentido, enquanto o impacto negativo de uma maior participação dos salários sobre a rentabilidade dos negócios não é muito grande, pode-se argumentar que não há, necessariamente, antagonismo entre capitalistas e trabalhadores, pois é possível aumentar, simultaneamente, os salários reais e o emprego de um lado, e as vendas e os lucros de outro. Porém, se o conflito entre capitalistas e trabalhadores levar a uma perda de competitividade externa, então o resultado final será uma forte restrição ao crescimento econômico do lado da demanda, e essa restrição será a clássica restrição do Balanço de Pagamentos.

Dessa discussão, o presente artigo tem como objetivo apresentar um modelo macrodinâmico de inspiração pós-keynesiana a fim de analisar os efeitos da abertura da conta de capitais do balanço de pagamentos sobre a performance macroeconômica (produto real, taxa de juros, taxa de inflação e regime de crescimento - wage-led ou profit-led) das economias em desenvolvimento.

O modelo aqui apresentado considera uma economia que possui as seguintes características: (i) regime de câmbio flutuante; (ii) empresas oligopolistas que fixam os preços com base em um mark-up fixo sobre os custos diretos unitários de 
produção ${ }^{2}$; (iii) os capitalistas domésticos têm acesso ao mercado internacional de capitais para financiarem as suas decisões de consumo e de investimento por intermédio da emissão de títulos privados denominados em moeda estrangeira.

O modelo em consideração será usado para analisar a sensibilidade da economia frente a choques exógenos como, por exemplo, variações nas taxas de juros doméstica e internacional, aumento do endividamento externo como proporção do estoque de capital e alterações na participação dos lucros na renda. Os exercícios de estática comparativa mostram que um aumento do endividamento externo pode ter efeitos benéficos para a economia no curto prazo, uma vez que induz um aumento do valor de equilíbrio de curto prazo do grau de utilização da capacidade produtiva e da taxa de crescimento do estoque de capital. Contudo, para identificar qual o efeito final da depreciação cambial sobre a economia, será necessário encontrar qual o regime de crescimento existente na economia (wage-led ou profit-led). Em particular, demonstrar-se-á que este regime depende, em última análise, da magnitude das elasticidades renda das importações e das exportações. Ou, de outra forma, que o regime wage-led está condicionado à superação da crônica restrição do balanço de pagamentos.

Por sua vez, a dinâmica de longo prazo do modelo mostra que, sob certas condições, o acesso ao mercado internacional de capitais pode gerar trajetórias explosivas para o sistema econômico, notadamente em um contexto em que a política monetária é conduzida com base em um regime estrito de metas de inflação. Além disso, demonstrar-se-á que uma redução da meta de inflação perseguida pelas autoridades monetárias em um contexto de abertura da conta de capitais do balanço de pagamentos pode gerar um aumento permanente da taxa real de juros - com reflexos negativos sobre a acumulação de capital - bem como um aumento do endividamento externo da economia, contribuindo assim para uma maior fragilidade externa.

O presente artigo se divide em seis seções incluindo a presente introdução. Na seção 1, descreve-se a tecnologia empregada pelas firmas desta economia. A seção 2 apresenta a fixação de preços por parte das empresas e a determinação da distribuição de renda entre salários e lucros. A seção 3 se preocupa com os determinantes da demanda efetiva. A seção 4 está dedicada à análise do equilíbrio de curto prazo do modelo. A seção 5 apresenta a dinâmica de longo prazo do modelo, e a última seção sumariza as conclusões obtidas ao longo do artigo.

(2) O ajuste entre poupança e investimento é feito por intermédio de variações no grau de utilização da capacidade produtiva. 


\section{A tecnologia}

As firmas produzem um bem homogêneo que é destinado tanto para consumo quanto para investimento. A produção de bens e serviços é feita com base em uma tecnologia de produção de coeficientes fixos à la Leontieff, dada pela seguinte equação:

$$
X=\min \left[\frac{L}{b} ; K u\right]
$$

Onde: $L$ é a quantidade de trabalho empregada na economia; $b$ é o requisito unitário de mão de obra; $X$ é o produto real; $K$ é o estoque de capital; $u$ é a relação produtocapital, uma proxy do grau de utilização da capacidade produtiva.

Seguindo a tradição pós-keynesiana, supõe-se uma economia na qual as firmas operam com capacidade produtiva excedente de forma que toda a variação da demanda agregada é atendida por intermédio de variações do grau de utilização da capacidade existente. Dessa forma, a demanda de trabalho por parte das firmas será dada pela seguinte expressão:

$$
L=b X
$$

\section{Formação de preços e distribuição de renda}

As firmas dessa economia operam em uma estrutura de mercado oligopolista, de tal maneira que elas têm poder de fixação de preços. Ademais, as empresas fixam os preços de seus produtos com base em um mark-up fixo sobre os custos diretos de produção. Tal como em Kalecki (1954), a distribuição da renda entre salários e lucros é determinada pela política de formação de preços das firmas, ou seja, pelas suas decisões a respeito do nível da taxa de mark-up sobre os custos diretos de produção.

Assim, o mark-up reflete o "grau de monopólio" da economia, o qual depende positivamente do grau de concentração industrial e inversamente do poder dos sindicatos (que são tomados exogenamente e não são explicitamente modelados aqui). Contudo, por se tratar de uma economia aberta, o mark-up também é influenciado pelo grau de competição dos produtos nacionais frente aos estrangeiros, de tal sorte que a dinâmica de preço dos produtos importados confere um limitador natural à expansão do mark-up.

Supondo que o trabalho e os insumos importados são os únicos custos diretos de produção, então as empresas irão fixar os preços dos seus produtos com base na seguinte equação:

$$
P=(1+\tau)\left[w b+m e P^{*}\right]
$$


Onde: $\tau$ é o mark-up; $w$ é o salário nominal; $m$ é requisito unitário de matérias primas importadas por unidade de produto $^{3}, e$ é a taxa nominal de câmbio, e $P^{*}$ é o preço do bem internacional.

Os lucros são determinados com base na diferença entre o valor nominal da produção e os custos com salários e insumos importados:

$$
P \Pi=P X-w b X-m e P{ }^{*} X
$$

Onde: $\Pi$ é o montante de lucros em termos reais; $P X$ é o valor nominal da produção; $w b X$ é a folha de salários e $m e P^{*} X$ é o valor nominal em moeda doméstica dos insumos importados.

Sendo assim, a participação dos lucros na renda é dada por ${ }^{4}$ :

$$
\pi=\frac{\tau}{1+\tau}
$$

A equação (5) reproduz um dos resultados básicos da teoria kaleckiana, segundo a qual a participação dos lucros na renda é determinada, em nível microeconômico, pelo grau de monopólio das firmas (Kalecki, 1971).

A taxa de lucro é obtida ao se dividir (4) por $P K$ :

$$
r=\frac{X}{P K}\left[P-w b-m e P^{*} X\right]
$$

Substituindo (2) e (3) em (6), obtém-se:

$$
r=\frac{\tau}{1+\tau} u \text { ou } \quad r=\pi . u
$$

Com base na equação (7), pode-se observar que a taxa de lucro depende apenas da participação dos lucros na renda e do grau de utilização da capacidade produtiva, tal como ocorre em uma economia fechada e sem governo ${ }^{5}$.

Dividindo-se a equação (3) por ( $p$ ), obtém-se a seguinte expressão:

(3) É importante observar que: $m=\frac{\mathrm{M}}{X}$

(4) A participação dos lucros na renda é definida como: $\pi=\frac{P X-w b X-m e P^{*} X}{P X}$. Temos então que: $\pi=\frac{P-w b-m e P^{*}}{P}=\frac{(1+\tau)\left[w b+m e P^{*}\right]-w b-m e P^{*}}{(1+\tau)\left[w b+m e P^{*}\right]}=\frac{\tau}{(1+\tau)}$.

(5) Ver Taylor (1991). 


$$
V=\frac{1}{b}[(1-\pi)-q m]
$$

Onde: $V=\frac{W}{P}$ é a taxa de salário real.

Sendo assim, a distribuição de renda nessa economia envolve três variáveis fundamentais, a saber: o salário real, a taxa real de câmbio e a participação dos lucros na renda. A participação dos lucros na renda é determinada pela equação (5), a partir de decisões microeconômicas a respeito da fixação da taxa de mark-up. Sendo assim, tem-se, ainda, duas variáveis endógenas a serem determinadas por intermédio da equação (8), a taxa de salário real e a taxa real de câmbio, fazendo com que a distribuição de renda esteja indeterminada.

Para eliminar a indeterminação na distribuição de renda, toma-se por hipótese que essa economia possui um setor de subsistência ou tradicional que funciona como uma reserva de mão de obra para as empresas que operam no setor capitalista $^{6}$. A existência desse excesso estrutural de força de trabalho faz com que, do ponto de vista do setor capitalista, a oferta de trabalho seja infinitamente elástica a um dado nível de salário real, o qual é tido como exógeno e determinado pelas convenções sociais existentes na economia em um determinado momento de sua história (cf. Bortis, 1997). Dessa forma, segue-se que:

$$
V=\bar{V}
$$

Assim, para cada nível de câmbio real, existe um equivalente nível de salário real $q=q(\bar{V})$. Isto significa, que variações na taxa real de câmbio proporcionam alterações no salário real, no mark-up das firmas e na distribuição funcional da renda.

\section{Demanda efetiva, inflação e conflito distributivo}

A economia em consideração possui relações com o exterior e também possui atividades governamentais. No entanto, por simplicidade, supõe-se que o governo não conduz nenhum tipo de atividade fiscal, de forma que o único instrumento ativo de política macroeconômica é a política monetária. A política monetária é conduzida por intermédio de operações de mercado aberto com vistas à fixação da taxa básica de juros.

A abertura da economia abrange três noções distintas, a saber: (i) a abertura do mercado de bens, entendida como a possibilidade de que as empresas e consumidores escolham entre bens nacionais e importados; (ii) a abertura dos mercados financeiros, entendido como a possibilidade que os agentes domésticos

(6) Essa mesma estratégia de eliminação de indeterminação de modelos macroeconômicos é adotada por Dutt (1990). 
escolham entre ativos financeiros nacionais e estrangeiros; (iii) a abertura do mercado de fatores, entendido como a possibilidade das empresas escolherem a localização de suas plantas produtivas em torno do mundo e a liberdade dos trabalhadores de migrarem livremente para outros países (Blanchard, 2001, p. 375376). No modelo aqui apresentado, contudo, trabalha apenas com os dois primeiros conceitos de abertura econômica.

Seguindo Bertella e Lima (2001), supõe-se que as transações comerciais das firmas com o exterior são determinadas pelas funções de exportação e importação (normalizadas em termos do estoque de capital), dadas por:

$$
\begin{aligned}
& \frac{\mathrm{E}}{K}=\varepsilon=\varepsilon_{0}+\varepsilon_{1} q-\varepsilon_{2} u+\varepsilon_{3} u^{*} \\
& \frac{\mathrm{M}}{K}=M=M_{0}-M_{1} q+M_{2} u
\end{aligned}
$$

Onde: $\varepsilon_{I}, M_{I}$ são parâmetros positivos; $q$ é a taxa real de câmbio e $u^{*}$ é o grau de utilização da capacidade produtiva das firmas que operam no exterior.

Pelas equações (10) e (11), verifica-se a validade da chamada condição de Marshall-Lerner, segundo a qual a depreciação da taxa real de câmbio gera um aumento das exportações líquidas. Por sua vez, a taxa de câmbio real é dada por:

$$
q=\frac{e P^{*}}{P}
$$

Onde: $e$ é a taxa de câmbio nominal; $P^{*}$ é o nível de preços dos bens importados em moeda estrangeira e $P$ é o nível de preços dos bens domésticos em moeda doméstica.

Substituindo (9) em (8), obtém-se a seguinte expressão:

$$
q=\frac{1}{m}[(1-\pi)-V \bar{b}]
$$

Dividindo-se a equação (11) por $u$ e substituindo a resultante em (13), encontra-se, após os algebrismos necessários, a seguinte equação:

$$
u=\frac{M_{1} q^{2}-M_{0} q}{M_{2} q-(1-\pi)+\overline{-} b}
$$

A equação (14) descreve o Lócus Distributivo ( $L D)$, definido como o conjunto das combinações entre o grau de utilização da capacidade produtiva e taxa real de câmbio $(u, q)$ para o qual há equilíbrio distributivo na economia; isto é, uma situação na qual o mark-up efetivo é igual ao desejado, o salário real efetivo é igual 
ao nível determinado pelas convenções sociais prevalecentes na economia e a taxa real de câmbio é aquela permitida pela equação (8).

Diferenciando (14) com respeito à $(u)$ e $(q)$, obtemos a expressão (15) que apresenta a inclinação do Lócus LD:

$$
\frac{\partial u}{\partial q} \mid L D=\frac{\left(M_{0}-2 M_{1} q\right)(1-\pi-\bar{V} b)+M_{1} M_{0} q^{2}}{\left[M_{2} q-(1-\pi-\bar{V} b)\right]^{2}}
$$

Com base em (15), constata-se que a inclinação do lócus $L D$ depende do valor assumido pela taxa real de câmbio. Com efeito, se $q>\frac{M_{0}}{2 M_{1}} \Rightarrow \frac{\partial u}{\partial q}<0$, caso contrário tem-se que $\frac{\partial u}{\partial q}>0$

Seguindo Robinson (1962) e Taylor (1985), supõe-se que a taxa desejada de crescimento do estoque de capital por parte das firmas depende de dois componentes. Um componente autônomo que capta o "otimismo espontâneo" dos capitalistas, ou seja, o seu "animal spirit" e outro componente que depende da diferença entre a taxa de retorno do capital e a taxa real de juros. Dessa forma, a função investimento é descrita por:

$$
\frac{I}{K}=g^{i}=g_{0}+h[r-i-\hat{P}]
$$

Onde: I é o investimento agregado; $g^{i}$ é a taxa desejada de crescimento do estoque de capital; go representa o "animal spirit" dos capitalistas; $h$ mede a sensibilidade da taxa desejada de crescimento do estoque de capital às diferenças entre a taxa de lucro e a taxa real de juros, $i$ é a taxa nominal de juros e $\hat{P}$ é a taxa corrente de inflação.

Tal como Rowthorn (1977), a inflação advém do conflito distributivo entre o capital e o trabalho. A inflação salarial irá ocorrer toda vez que os trabalhadores desejarem uma participação dos salários (dos lucros) na renda nacional maior (menor) do que o valor corrente dessa variável. Dessa forma, a equação de movimento que descreve a inflação salarial é representada por intermédio de:

$$
\hat{w}=\psi\left[\pi-\pi^{F}\right]
$$


Onde: $w$ é a taxa proporcional de variação do salário nominal; $\psi$ indica a velocidade de ajustamento, sendo que $0<\psi<1 ; \pi^{F}$ é a participação nos lucros na renda que é desejada pelos trabalhadores.

A participação dos lucros na renda desejada pelos trabalhadores depende claramente do poder de barganha desses trabalhadores no mercado de trabalho. Dessa forma, o aumento do nível de emprego aumenta o poder de barganha dos trabalhadores e, portanto, evidencia-se maior pressão sobre a parcela da renda que eles desejam apropriar. Como, pela equação (2), o emprego depende do nível de produção, segue-se que o grau de utilização da capacidade produtiva pode ser utilizado como uma proxy para o nível de emprego. Sendo assim, tem-se que:

$$
\pi^{F}=\alpha_{0}-\alpha_{1} u
$$

Como o salário real desejado pelos trabalhadores é, por hipótese, constante, função das convenções sociais, segue-se que a inflação salarial é instantaneamente repassada para os preços. Sendo assim, tem-se que:

$$
\hat{P}=\hat{w}
$$

Substituindo (18) em (17) e a resultante em (19), obtemos a seguinte equação:

$$
\hat{P}=\psi \pi-\psi \alpha_{0}+\psi \alpha_{1} u
$$

A equação (20) é uma espécie de curva de Phillips para a economia em consideração na medida em que estabelece uma relação inversa entre a taxa de inflação e o grau de utilização da capacidade produtiva.

Substituindo (20) em (16), obtém-se a expressão para a taxa desejada de crescimento do estoque de capital:

$$
g^{i}=g_{0}-h \psi \alpha_{0}+h\left[\pi-\psi \alpha_{1}\right] u-h i-h \psi \pi
$$

Seguindo a tradição de Marx (1988), Kalecki (1954, 1971), Kaldor (1956, 1957), Robinson (1956, 1962) e Pasinetti (1962), supõe-se que os capitalistas recebem, sob a forma de lucros, todo o excedente sobre os salários, poupando uma fração constante de sua renda $\left(s_{\pi}\right)$. Já os trabalhadores gastam toda a sua renda com consumo.

Deve-se ressaltar, contudo, que uma parte da renda dos capitalistas domésticos não está disponível para o financiamento dos seus gastos de consumo, pois é remetida para o exterior na forma de encargos financeiros sobre a dívida 
externa do setor privado. Dessa forma, a poupança dos capitalistas pode ser apresentada pela seguinte equação:

$$
P S=s_{\pi}\left(r P K-e i^{*} B\right)
$$

Onde: $S$ é a poupança dos capitalistas; $s_{\pi}$ é a propensão a poupar a partir dos lucros; $i^{*}$ é a taxa de juros dos títulos internacionais e $B$ é o estoque da dívida externa do setor privado.

No que se refere ao financiamento externo, apenas as firmas que possuem um valor mínimo de capital e lucros estão dispostas e capazes de demandar poupança externa para complementar a sua própria acumulação interna de capital. Este financiamento é estritamente privado e realizado por intermédio de emissão de títulos à taxa de juros $\left(i^{*}\right)$.

Nesse contexto, é importante destacar que esta economia opera em um regime de mobilidade imperfeita de capitais com câmbio flutuante no sentido de Mundell (1962) e Fleming (1962); de tal forma que a existência de ganhos de arbitragem entre títulos domésticos e os títulos internacionais dá origem a um fluxo de entrada/saída de capitais finito por unidade de tempo. Isso porque supõe-se que os títulos doméstico e internacional são substitutos imperfeitos entre si. Dessa forma, não vale a equação de paridade descoberta da taxa de juros. Além disso, a hipótese de pequena economia aberta assegura que a taxa de juros doméstica não tem efeito sobre a taxa de juros internacional.

Dividindo-se (22) por ( $P K$ ) e após alguns algebrismos, encontra-se:

$$
g^{s}=s_{\pi} \pi u-\frac{s_{\pi} i^{*} Z}{P^{*}} q
$$

Onde: $g^{S}$ é a taxa de crescimento do estoque de capital permitida pela disponibilidade de poupança dessa economia e $Z$ é o endividamento externo como proporção do estoque de capital.

No curto prazo, admite-se que o estoque de capital ( $K$ ), o coeficiente unitário de mão de obra $(b)$ e o mark-up $(\tau)$ são constantes. Dessa forma, o grau de utilização da capacidade produtiva é a variável de ajuste entre a taxa de crescimento do estoque de capital que é desejada pelas firmas e a taxa de crescimento do estoque de capital que é permitida pela disponibilidade de poupança da economia. A condição de equilíbrio macroeconômico é dada, então, por:

$$
g^{i}=g^{S}-(\varepsilon-M)
$$

Substituindo (10), (11), (21) e (23) em (24) e resolvendo para ( $u$ ), obtémse, após os necessários algebrismos, a seguinte expressão: 


$$
u=\frac{1}{s_{\pi} \pi-h\left[\pi-\psi \alpha_{1}\right]+\varepsilon_{2}+M_{2}}\left[\left(g_{0}+h \psi \alpha_{0}+\varepsilon_{0}-M_{0}\right)-h i-h \psi \pi+\left(\frac{s_{\pi} i^{*} Z}{P^{*}}+\varepsilon_{1}+M_{1}\right) q\right]
$$

A equação (25) descreve o Lócus IS, definido como sendo o conjunto das combinações entre grau de utilização da capacidade produtiva e câmbio real $(u, q)$, para os quais o mercado de bens está em equilíbrio.

Para obter a inclinação do Lócus IS basta diferenciar (25) com respeito a ( $u$ ) e ( $q)$, de forma a se obter a seguinte expressão:

$$
\frac{\partial u}{\partial q} \mid I S=\frac{1}{s_{\pi} \pi-h\left[\pi-\psi \alpha_{1}\right]+\varepsilon_{2}+M_{2}}\left[\frac{s_{\pi} i^{*} Z}{P^{*}}+\varepsilon_{1}+M_{1}\right]>0
$$

Em palavras: uma depreciação da taxa real de câmbio gera um aumento do grau de utilização da capacidade produtiva. Isso decorre tanto do efeito positivo que a desvalorização da taxa real de câmbio tem sobre as exportações líquidas (dado que estamos supondo a validade da condição Marshall-Lerner) como também do fato que uma desvalorização da taxa real de câmbio, ao aumentar a renda enviada para o exterior (devido aos encargos financeiros da dívida externa privada), reduz a poupança dos capitalistas domésticos. Dessa forma, o ajuste no mercado de bens exige um aumento do grau de utilização da capacidade produtiva.

Diferenciando (25) com respeito a $Z$, obtemos:

$$
\frac{\partial u}{\partial Z}=\frac{1}{s_{\pi} \pi-h\left[\pi-\psi \alpha_{1}\right]+\varepsilon_{2}+M_{2}}\left(\frac{s_{\pi} i^{*}}{P^{*}}\right)>0
$$

Em outras palavras, um aumento do endividamento externo (como proporção do estoque de capital) irá aumentar o grau de utilização da capacidade produtiva. Isso porque um aumento do endividamento externo reduz a renda disponível dos capitalistas (pós-pagamento dos encargos da dívida externa privada), levando-os a reduzir a sua poupança. Dessa forma, o ajuste no mercado de bens também termina por exigir um aumento do nível de utilização da capacidade produtiva.

\section{A configuração de equilíbrio de curto prazo e a estática comparativa}

A economia estará em equilíbrio de curto prazo quando o grau de utilização da capacidade produtiva $(u)$ e a taxa real de câmbio forem tais a satisfazer simultaneamente a equação (14) - referente ao lócus LD - e a equação (25) referente ao lócus IS. Como a inclinação do Lócus LD muda quando a taxa real de câmbio ultrapassa um certo valor crítico dado por $q_{c}=\frac{M_{0}}{2 M_{1}}$; segue-se que existem 
pelo menos duas configurações possíveis para o equilíbrio de curto prazo, tal como pode ser observado na Figura 1 abaixo:

\section{Figura 1}

Possíveis configurações de equilíbrio de curto prazo

\section{Caso A}

u

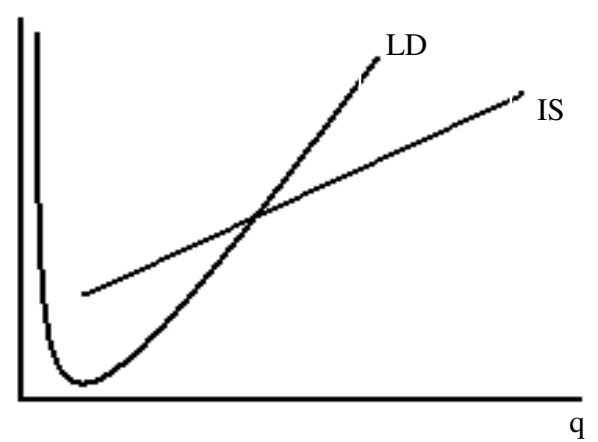

Caso B

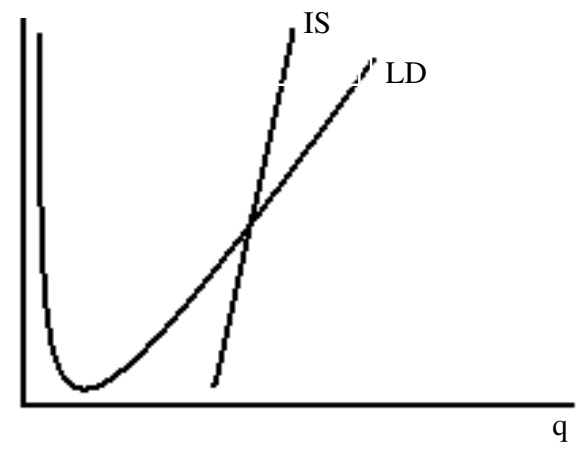

Pode-se demonstrar que apenas a situação representada pelo caso A referese a um equilíbrio de curto prazo estável. Dessa forma, supõe-se que: $\frac{\partial u}{\partial q}\left|L D>\frac{\partial u}{\partial q}\right| I S$

Para encontrar a taxa real de câmbio de equilíbrio de curto prazo do sistema, iguala-se a equação (14) com a equação (25). Dessa forma, encontra-se a seguinte expressão:

$\frac{M_{1} q^{2}-M_{0} q}{M_{2} q-(1-\pi-\bar{V} b)}-\frac{1}{s_{\pi} \pi-h\left[\pi-\psi \alpha_{1}\right]+\varepsilon_{2}+M_{2}}\left[\left(g_{0}+h \psi \alpha_{0}+\varepsilon_{0}-M_{0}\right)-h i-h \psi \pi\right.$ $\left.+\left(\frac{s_{\pi} i^{*} Z}{P^{*}}+\varepsilon_{1}+M_{1}\right) q+\varepsilon_{3}\right]=0$

A expressão (27) é utilizada para calcular os efeitos de mudanças exógenas da taxa de juros internacional, da taxa de juros doméstica, da dívida externa como proporção do estoque de capital e da participação dos lucros na renda sobre o valor de equilíbrio de curto prazo da taxa real de câmbio. Diferenciando (27) com respeito a $i, i^{*}, Z \quad e \quad \pi$, tem-se:

$$
\frac{\partial q}{\partial i}=-\frac{\varphi_{2}}{\varphi_{1}}<0
$$




$$
\begin{gathered}
\frac{\partial q}{\partial Z}=-\frac{\varphi_{3}}{\varphi_{1}}>0 \\
\frac{\partial q}{\partial i^{*}}=-\frac{\varphi_{4}}{\varphi_{1}}>0 \\
\frac{\partial q}{\partial \pi}=-\frac{\varphi_{5}}{\varphi_{1}}=?
\end{gathered}
$$

Onde:

$$
\begin{aligned}
& \frac{\left(M_{0}-2 M_{1} q\right)(1-\pi-\bar{V} b)+M_{1} M_{0} q^{2}}{\left[M_{2} q-(1-\pi-\bar{V} b)\right]^{2}}-\frac{1}{s_{\pi} \pi-h\left[\pi-\psi \alpha_{1}\right]+\varepsilon_{2}+M_{2}}\left[\frac{s_{\pi} i^{*} Z}{P^{*}}+\varepsilon_{1}+M_{1}\right]=\varphi_{1}>0 \\
& \frac{h}{s_{\pi} \pi-h\left[\pi-\psi \alpha_{1}\right]+\varepsilon_{2}+M_{2}}=\varphi_{2}>0 \\
& -\frac{s_{\pi} q i^{*}}{\left[s_{\pi} \pi-h\left[\pi-\psi \alpha_{1}\right]+\varepsilon_{2}+M_{2}\right] P^{*}}=\varphi_{3}<0 \\
& -\frac{s_{\pi} q Z}{\left[s_{\pi} \pi-h\left[\pi-\psi \alpha_{1}\right]+\varepsilon_{2}+M_{2}\right] P^{*}}=\varphi_{4}<0 \\
& \frac{g_{0}+h \psi \alpha_{0}+\varepsilon_{0}-M_{0}-h[i+\psi \pi]+\left[\frac{s_{\pi^{i}} Z}{P^{*}}+\varepsilon_{1}+M_{1}\right] q+u^{*}}{\left[s_{\pi} \pi-h\left[\pi-\psi \alpha_{1}\right]+\varepsilon_{2}+M_{2}\right]^{2}}\left(s_{\pi}-h\right)+\frac{h \psi}{s_{\pi} \pi-h\left[\pi-\psi \alpha_{1}\right]+\varepsilon_{2}+M_{2}}
\end{aligned}
$$

A expressão (28a) diz que um aumento da taxa de juros doméstica gera uma apreciação do câmbio real. Com efeito, um aumento da taxa de juros doméstica irá reduzir a taxa de crescimento do estoque de capital que é desejada pelas firmas, deslocando para baixo a curva IS. Dada a distribuição de renda, tem-se um aumento do requisito de matéria-prima importada por unidade de produto, de tal forma que a taxa real de câmbio se aprecia.

A expressão (28b) mostra que um aumento do endividamento externo como proporção do estoque de capital gera uma depreciação da taxa de câmbio real de equilíbrio. Isto porque um aumento do endividamento externo irá resultar em um aumento da renda que os capitalistas domésticos têm que remeter para o exterior na forma de encargos financeiros (juros + amortizações). Dessa forma, ocorre uma redução da poupança dos capitalistas e, portanto, um aumento dos seus gastos de consumo. Esse aumento do consumo desloca a curva IS para cima, de tal forma que o grau de utilização da capacidade produtiva aumenta. Dada a distribuição de renda 
entre salários e lucros, o requisito de matéria-prima importada por unidade de produto se reduz, depreciando, portanto, o câmbio real.

Dada a ambiguidade no coeficiente de $\left(\varphi_{5}\right)$, algumas considerações se fazem necessárias. No curto prazo, o efeito do aumento/redução dos lucros sobre o câmbio real dependerá da elasticidade das exportações e importações com respeito à taxa de câmbio real ${ }^{7}$. Isto porque, dada a validade da condição de Marshall-Lerner, a resposta da balança comercial em relação a mudanças na distribuição funcional de renda é lenta de tal forma que, por exemplo, a elevação dos lucros na renda aumenta o consumo de produtos importados, o que se traduz em uma piora da balança comercial.

Por outro lado, esta mudança na balança comercial pode se traduzir em uma redução do investimento privado no curto prazo em razão da perda de competitividade externa, o que reduziria o grau de utilização da capacidade produtiva. Acrescenta-se que, o aumento da participação dos lucros na renda reduz a participação relativa dos salários no produto, que deve cair ainda mais com a depreciação da moeda, o que intensifica a queda da demanda e do produto.

No longo prazo, por sua vez, sabe-se que uma depreciação/apreciação da moeda é equivalente a uma redução/elevação dos salários reais (cf. Lópes, 2001, p. 297). Assim, aumentar a participação dos salários na renda, ou adotar o regime wage-led, é equivalente a adotar uma estratégia de apreciação da taxa de câmbio. Por sua vez, crescer com aumento da participação dos lucros, por meio da estratégia profit-led, só é possível se esse expediente for acompanhado da depreciação da taxa de câmbio.

Desta forma, se uma economia for do tipo wage-led, a depreciação cambial tende a gerar efeitos contracionistas sobre a produção e emprego, mas é relativamente eficaz para melhorar as transações correntes. Por outro lado, se uma economia for do tipo profit-led, a desvalorização cambial tende a gerar efeitos expansionistas sobre a economia, mas é menos eficaz para melhorar as transações correntes. Contudo, a consistência da estratégia de crescimento depende essencialmente dos parâmetros estruturais relacionados às elasticidades-renda das exportações $\left(\varepsilon_{2}\right)$ e importações $\left(M_{2}\right)$, conforme exposto no Quadro 1 a seguir:

(7) Se as firmas exportadoras não estiverem dispostas a reduzirem seus preços no mercado internacional, dado o seu poder de monopólio, então a demanda por exportações não será afetada de forma substancial e o efeito final da balança comercial dependerá da elasticidade-preço das exportações. 
Quadro 1

Parâmetros Estruturais e estratégias de crescimento

\begin{tabular}{|c|c|c|}
\hline Parâmetros Estruturais & Regime de Crescimento & $\begin{array}{c}\text { Efeitos Macroeconômicos do } \\
\text { aumento da participação dos } \\
\text { lucros na renda }\end{array}$ \\
\hline $\begin{array}{l}\text { Elasticidade-renda das } \\
\text { exportações maior do que } \\
\text { Elasticidade-renda das } \\
\text { importações } \\
\text { "Economias Desenvolvidas" }\end{array}$ & wage-led-growth & $\begin{array}{l}\frac{\partial q}{\partial \pi}<0 \\
\frac{\partial u}{\partial \pi}<0 \\
\frac{\partial g}{\partial \pi}<0 \\
\frac{\partial \hat{P}}{\partial \pi}<0\end{array}$ \\
\hline $\begin{array}{l}\text { Elasticidade-renda das } \\
\text { exportações menor do que } \\
\text { Elasticidade-renda das } \\
\text { importações }\end{array}$ & 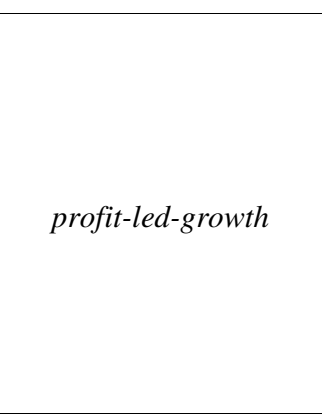 & $\begin{array}{l}\frac{\partial q}{\partial \pi}>0 \\
\frac{\partial u}{\partial \pi}>0 \\
\frac{\partial g}{\partial \pi}>0 \\
\frac{\partial \hat{P}}{\partial \pi}>0\end{array}$ \\
\hline
\end{tabular}

No geral, seguindo os resultados clássicos da Lei de Thirlwall, a taxa de crescimento do produto de um país depende da elasticidade-renda da demanda de importação, ou mais precisamente, depende da sensibilidade com que as exportações de um país reagem às variações da renda do resto do mundo. Assim, para economias abertas desenvolvidas, o modelo de crescimento wage-led é perfeitamente plausível, porque uma expansão dos salários na renda é acompanhada de aumento do grau de utilização da capacidade produtiva e do crescimento, observado o tradicional tradeoff entre crescimento e inflação. Já para os países em desenvolvimento, a restrição a essa estratégia reside nos problemas estruturais de balanço de pagamentos. Nesse sentido, argumenta-se que a estratégia ideal para esses países é o regime do tipo profit-led, dado a relação inversa entre crescimento dos salários e câmbio real.

É importante salientar que os segmentos mais pobres da população são os que mais sofrem com a queda no produto e no emprego. Assim, a redução da participação dos salários na renda não significa piora das condições de vida. Ao contrário, a dinâmica da distribuição funcional da renda mostra qual a estratégia possível de crescimento de longo prazo consistente com a geração de emprego e renda. Como resultado final desta discussão, admite-se que $\frac{\partial q}{\partial \pi}>0$, uma vez que o 
foco da análise é a estratégia de crescimento dos países em desenvolvimento com desemprego estrutural.

Os efeitos de variações da taxa doméstica de juros, da taxa de juros internacional, da dívida externa como proporção do estoque de capital e da participação dos lucros na renda podem ser analisados por intermédio das seguintes derivadas parciais ${ }^{8}$ :

$$
\begin{gathered}
\frac{\partial u}{\partial i}=-\frac{\theta_{2}}{\theta_{1}}<0 \\
\frac{\partial u}{\partial Z}=-\frac{\theta_{3}}{\theta_{1}}>0 \\
\frac{\partial u}{\partial i^{*}}=-\frac{\theta_{4}}{\theta_{1}}>0 \\
\frac{\partial u}{\partial \pi}=-\frac{\theta_{5}}{\theta_{1}}>0
\end{gathered}
$$

Onde:

$$
\begin{aligned}
& \frac{M_{2}\left[\left(u M_{2}+M_{0}\right)^{2}-4 M_{1} u(1-\pi-\bar{V} b)\right]^{1 / 2}+M_{2}\left(u M_{2}+M_{0}\right)-2 M_{1}(1-\pi-\bar{V} b)}{2 M_{1}\left[\left(u M_{2}+M_{0}\right)^{2}-4 M_{1} u(1-\pi-\bar{V} b)\right]^{1 / 2}}-\frac{s_{\pi} \pi-h\left[\pi-\psi \alpha_{1}\right]+\varepsilon_{2}+M_{2}}{\left[\frac{s_{\pi} i^{*} Z}{P^{*}}+\varepsilon_{1}+M_{1}\right]}=\theta_{1}<0 \\
& -\frac{h}{\left[\frac{s_{\pi} i^{*} Z}{P^{*}}+\varepsilon_{1}+M_{1}\right]}=\theta_{2}<0 \\
& \frac{s_{\pi} i^{*}\left[u\left[s_{\pi} \pi-h\left[\pi-\psi \alpha_{1}\right]+\varepsilon_{2}+M_{2}\right]-\left(g_{0}+h \psi \alpha_{0}+\varepsilon_{0}-M_{0}\right)+h i+h \psi \pi-u^{*}\right]}{P^{*}\left[\frac{s_{\pi} i^{*} Z}{P^{*}}+\varepsilon_{1}+M_{1}\right]^{2}}=\theta_{3}>0 \\
& \frac{s_{\pi} Z\left[u\left[s_{\pi} \pi-h\left[\pi-\psi \alpha_{1}\right]+\varepsilon_{2}+M_{2}\right]-\left(g_{0}+h \psi \alpha_{0}+\varepsilon_{0}-M_{0}\right)+h i+h \psi \pi-u^{*}\right]}{P^{*}\left[\frac{s_{\pi} i^{*} Z}{P^{*}}+\varepsilon_{1}+M_{1}\right]^{2}}=\theta_{4}<0 \\
& -\frac{u\left(s_{\pi}-h\right)+h \psi}{\left[\frac{s_{\pi} i^{*} Z}{P^{*}}+\varepsilon_{1}+M_{1}\right]}=\theta_{5}<0
\end{aligned}
$$

(8) Essas expressões podem ser obtidas ao se substituir (27) em (14) e diferenciar a expressão resultante com respeito a $i, i *, Z$ e $\pi$. 
A expressão (29a) mostra que um aumento da taxa básica de juros provoca uma redução do grau de utilização da capacidade produtiva de equilíbrio de curto prazo, em razão da redução da taxa desejada de crescimento do estoque de capital.

A expressão (29b) mostra que um aumento da dívida externa (como proporção do estoque de capital) gera um aumento do grau de utilização da capacidade produtiva compatível com o equilíbrio de curto prazo do sistema econômico. Esse resultado é uma decorrência elementar do fato de que um aumento da dívida externa do setor privado gera uma expansão da demanda efetiva, tal como podemos observar na equação (26b). Isso equivale a um deslocamento para cima da curva IS na figura A. O lócus LD, por sua vez, não se altera com mudanças no endividamento externo como proporção do estoque de capital. Sendo assim, haverá um novo equilíbrio de curto prazo com um nível de utilização da capacidade produtiva mais alto (e uma taxa de câmbio mais depreciada).

A equação (29c) mostra que um aumento da taxa de juros internacional gera um aumento do grau de utilização da capacidade produtiva. Esse resultado pouco comum é fruto da hipótese aqui adotada a respeito do regime de mobilidade de capitais. Como a mobilidade de capitais é imperfeita no sentido de Mundell e Fleming, segue-se que um aumento da taxa de juros internacional não induz um aumento da taxa de juros doméstica. No entanto, o aumento da renda enviada para o exterior induz uma depreciação da taxa real de câmbio [ver equação 28(c)], o que aumenta as exportações líquidas, induzindo, dessa forma, uma expansão da demanda efetiva e do grau de utilização da capacidade produtiva de equilíbrio de curto prazo.

Por fim, a expressão (29d) mostra que um aumento da participação dos lucros na renda aumenta o grau de utilização da capacidade produtiva.

Com base na equação (21), sabemos que $\frac{\partial g^{I}}{\partial u}>0$. Dessa forma, pela regra da cadeia é possível afirmar que:

$$
\begin{gathered}
\frac{\partial g^{i}}{\partial i}=\frac{\partial g^{i}}{\partial u} \frac{\partial u}{\partial i}=(+)(-)<0 \\
\frac{\partial g^{i}}{\partial Z}=\frac{\partial g^{i}}{\partial u} \frac{\partial u}{\partial Z}=(+)(+)>0 \\
\frac{\partial g^{i}}{\partial i^{*}}=\frac{\partial g^{i}}{\partial u} \frac{\partial u}{\partial i^{*}}=(+)(+)>0 \\
\frac{\partial g^{i}}{\partial \pi}=\frac{\partial g^{i}}{\partial u} \frac{\partial u}{\partial \pi}=(+)(+)>0
\end{gathered}
$$


Em outras palavras, a taxa de crescimento do estoque de capital é uma função inversa da taxa nominal de juros doméstica e direta da participação dos lucros na renda. Dessa forma, pode-se afirmar que nessa economia prevalece um regime de crescimento do tipo profit-led, devido à clássica restrição de Balanço de Pagamentos derivada das elasticidades-renda das exportações e importações. Além disso, pode-se argumentar que a taxa de crescimento do estoque de capital é uma função direta do endividamento externo como proporção do estoque de capital e da taxa de juros internacional.

Da mesma forma, da equação (20) sabe-se que: $\frac{\partial \hat{P}}{\partial u}>0$. Logo, utilizando o mesmo expediente, tem-se que:

$$
\begin{gathered}
\frac{\partial \hat{P}}{\partial i}=\frac{\partial \hat{P}}{\partial u} \frac{\partial u}{\partial i}=(+)(-)<0 \\
\frac{\partial \hat{P}}{\partial Z}=\frac{\partial \hat{P}}{\partial u} \frac{\partial u}{\partial Z}=(+)(+)>0 \\
\frac{\partial \hat{P}}{\partial i^{*}}=\frac{\partial \hat{P}}{\partial u} \frac{\partial u}{\partial i^{*}}=(+)(+)>0 \\
\frac{\partial \hat{P}}{\partial \pi}=\frac{\partial \hat{P}}{\partial u} \frac{\partial u}{\partial \pi}=(+)(+)>0
\end{gathered}
$$

Pelas expressões (29a), (30a) e (31a), verifica-se que a política monetária é capaz de influenciar a performance de variáveis econômicas como inflação, grau de utilização da capacidade produtiva e taxa de crescimento do estoque de capital no contexto de uma economia aberta com mobilidade imperfeita de capitais. No entanto, se a autoridade monetária resolver reduzir a taxa de inflação, então este objetivo só poderá ser alcançado por intermédio de uma redução da taxa de crescimento. Dentro deste contexto, verifica-se claramente que os objetivos, no curto prazo, de crescimento e baixa inflação são conflitantes.

A expressão (30b) mostra que, no curto prazo, o acesso ao mercado internacional de capitais e o endividamento externo a ele correlato contribuem para aumentar a taxa de crescimento do estoque de capital.

Deve-se ressaltar que o modelo aqui desenvolvido não aborda questões importantes relacionadas ao aspecto da distribuição pessoal da renda, mas sim, sobre a distribuição funcional da renda. Sobre este tema, por exemplo, Carvalho e Rezai mostram as condições nas quais a redução da desigualdade de renda entre trabalhadores torna a demanda mais wage-led. Mais precisamente, os autores 
mostram que a economia pode se mover de uma situação wage-led para profit-led se a função de demanda agregada é uma função positiva da desigualdade de renda. Também nessa linha de investigação, Palley (2014) mostra que as características microeconômicas podem alterar endogenamente a participação dos salários na renda afetando, portando, o grau de utilização da capacidade produtiva.

\section{A dinâmica de longo prazo}

Para analisar a dinâmica de longo prazo dessa economia, é necessário explicitar as equações de movimento do modelo. Seguindo Simonsen e Cysne (1995), a equação diferencial que descreve a dinâmica temporal da dívida externa é dada por:

$$
\dot{Z}=i^{*} D-H
$$

Onde: $D$ é o estoque de dívida externa;

$H$ é a transferência líquida de recursos para o exterior.

Diferenciando $z$ com respeito ao tempo, obtém-se a seguinte expressão:

$\dot{Z}=\frac{\dot{D}}{H}-\frac{D}{K} g^{i}$

Substituindo (32) em (33), encontra-se:

$$
\dot{Z}=\left(i^{*}-g^{i}\right) Z-\frac{H}{K}
$$

Substituindo (26) em (34), obtém-se a equação que descreve a dinâmica temporal da dívida externa como proporção do estoque de capital:

$$
\dot{Z}=Z\left(h \psi \alpha_{0}-g_{0}\right)+i^{*} Z+h i Z+h \psi \pi Z-h Z\left(\pi-\psi \alpha_{1}\right) u(i, Z, \pi)-\frac{H}{K}
$$

Admite-se que, na economia em consideração, a política monetária é conduzida no contexto de um regime de metas de inflação no qual a autoridade monetária deve utilizar a taxa nominal de juros com o objetivo de obter uma meta numérica definida para a taxa de inflação no médio prazo. Dessa forma, a política monetária é conduzida com base em uma função de reação do Banco Central dada por:

$$
i=\phi\left[\hat{P}(i, Z, \pi)-\hat{P}^{*}\right]
$$

Onde: $\phi$ é um parâmetro positivo que representa a velocidade com a qual o Banco Central reage a desvios entre a taxa de inflação efetiva e a meta inflacionária e $\hat{P}^{*}$ é a meta de inflação perseguida pela autoridade monetária. 
As expressões (35) e (36) formam um sistema de equações diferenciais que determinam a dinâmica da dívida externa como proporção do estoque de capital e da taxa básica de juros da economia. A Matriz Jacobiana do sistema é dada por:

$$
\left[\begin{array}{c}
\dot{Z} \\
\dot{i}
\end{array}\right]=\left[\begin{array}{cc}
{\left[\left(h \psi a_{0}-g_{0}\right)+h i+i^{*}+h \psi \lambda t-h\left(\pi-\psi a_{1}\right)\left(u+Z \frac{\partial u}{\partial Z}\right)\right.} & h Z\left[1-\left(\pi-\psi a_{1}\right) \frac{\partial u}{\partial i}\right] \\
\phi \frac{\partial \hat{P}}{\partial Z} & \phi \frac{\partial \hat{P}}{\partial i}
\end{array}\right]\left[\begin{array}{c}
Z-Z_{0} \\
i-i_{0}
\end{array}\right]
$$

Defina-se:

$$
\begin{aligned}
& \lambda_{0}=\left[\left(h \psi a_{0}-g_{0}\right)+h i+i^{*}+h \psi \pi\right]-h\left(\pi-\psi a_{1}\right)\left(u+Z \frac{\partial u}{\partial Z}\right)=? ; \\
& \lambda_{1}=h Z\left[1-\left(\pi-\psi a_{1}\right) \frac{\partial u}{\partial i}\right]>0 ; \lambda_{2}=\phi \frac{\partial \hat{P}}{\partial Z}>0 ; \lambda_{3}=\phi \frac{\partial \hat{P}}{\partial i}<0
\end{aligned}
$$

Analisando o sinal dos elementos da matriz Jacobiana, a única ambiguidade refere-se ao sinal de $\lambda_{0}$ que pode ser positivo ou negativo.

Defina-se: $\frac{Z}{u} \frac{\partial u}{\partial Z}=\varepsilon_{Z}>0$ como sendo a elasticidade do grau de utilização da capacidade produtiva com respeito ao endividamento externo, uma medida da sensibilidade do nível de atividade econômica com respeito a variações do nível de endividamento externo do setor privado. Sendo assim, temos que:

$$
\lambda_{0}=\left[\left(h \psi a_{0}-g_{0}\right)+h i+i^{*}+h \psi \pi\right]-h\left(\pi-\psi a_{1}\right) u\left(1+\varepsilon_{z}\right)
$$

$\mathrm{Na}$ expressão acima observamos que o sinal de $\lambda_{0}$ depende do valor assumido por essa elasticidade. Em particular, se $\varepsilon_{z}<\frac{h \psi a_{0}-g_{0}+i^{*}+h \psi \pi}{h\left(\pi-\psi a_{1}\right) u}-1$, então $\lambda_{0}$ será positivo. No que se segue iremos, portanto, supor que a elasticidade do grau de utilização da capacidade produtiva com respeito ao endividamento externo é baixa.

Dessa forma, o determinante e o traço da matriz Jacobiana são dados por:

$$
\begin{aligned}
& \operatorname{Tr}(J)=\lambda_{0}+\lambda_{3}<0 \\
& \operatorname{Det}(J)=\left(\lambda_{0} \lambda_{3}\right)-\left(\lambda_{1} \lambda_{2}\right)<0
\end{aligned}
$$

Como o determinante da matriz Jacobiana é negativo, o equilíbrio de longo prazo do sistema econômico é instável do tipo trajetória de sela (cf. Takayama, 1993, 
p. 407-408). Esse resultado aponta para a ideia de que a abertura da conta de capitais do balanço de pagamentos, embora possa ter efeitos benéficos para a economia no curto prazo, pode ser fonte de instabilidade a longo prazo em consonância com a argumentação desenvolvida por Bresser e Nakano (2003).

Em steady-state, o endividamento externo e a taxa básica de juros são constantes ao longo do tempo. Isto permite com que o lócus $\dot{Z}=0$ e o lócus $\dot{i}=0$, cujas inclinações são dadas respectivamente pelas seguintes equações, sejam determinados:

$$
\begin{aligned}
& \left.\frac{\partial i}{\partial Z}\right|_{\dot{Z}=0}>0 \\
& \left.\frac{\partial i}{\partial Z}\right|_{i=0}<0
\end{aligned}
$$

Sendo assim, a configuração de equilíbrio de longo prazo da economia em consideração pode ser visualizada por meio da Figura 2.

Figura 2

Configuração de equilíbrio de longo prazo

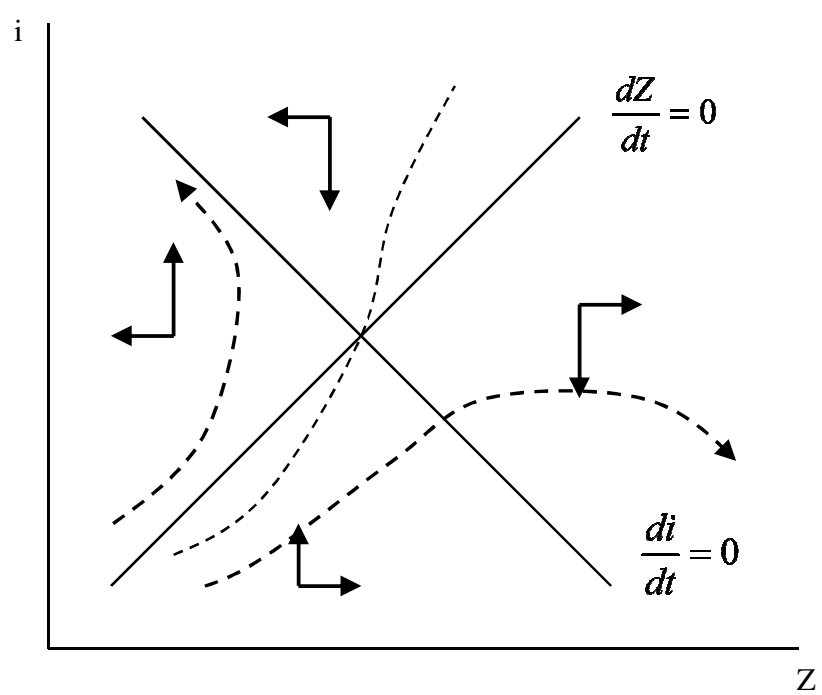

No que se segue, supõe-se que a autoridade monetária ajusta a taxa nominal de juros de maneira a garantir que a economia esteja sempre sobre a sua trajetória de sela.

Nesse contexto, suponha que as autoridades monetárias resolvam perseguir uma meta de inflação mais baixa. Qual seria o impacto sobre o equilíbrio de longo 
prazo do sistema econômico? Uma redução da meta de inflação irá deslocar o lócus $\frac{d i}{d t}=0$ para cima e para a direita, determinando um novo equilíbrio de longo prazo no qual a taxa nominal de juros e o endividamento externo como proporção do estoque de capital são ambos mais elevados (Figura 3). Como no equilíbrio de longo prazo a taxa de inflação efetiva deve ser igual à meta inflacionária, segue-se que a taxa efetiva de inflação irá se reduzir, impondo assim um aumento da taxa real de juros. Daqui se segue que uma redução da meta de inflação resulta em um aumento da taxa real de juros e do endividamento externo (como proporção do estoque de capital) de equilíbrio de longo prazo do sistema econômico.

Figura 3

Efeitos de longo prazo de uma redução da meta de inflação

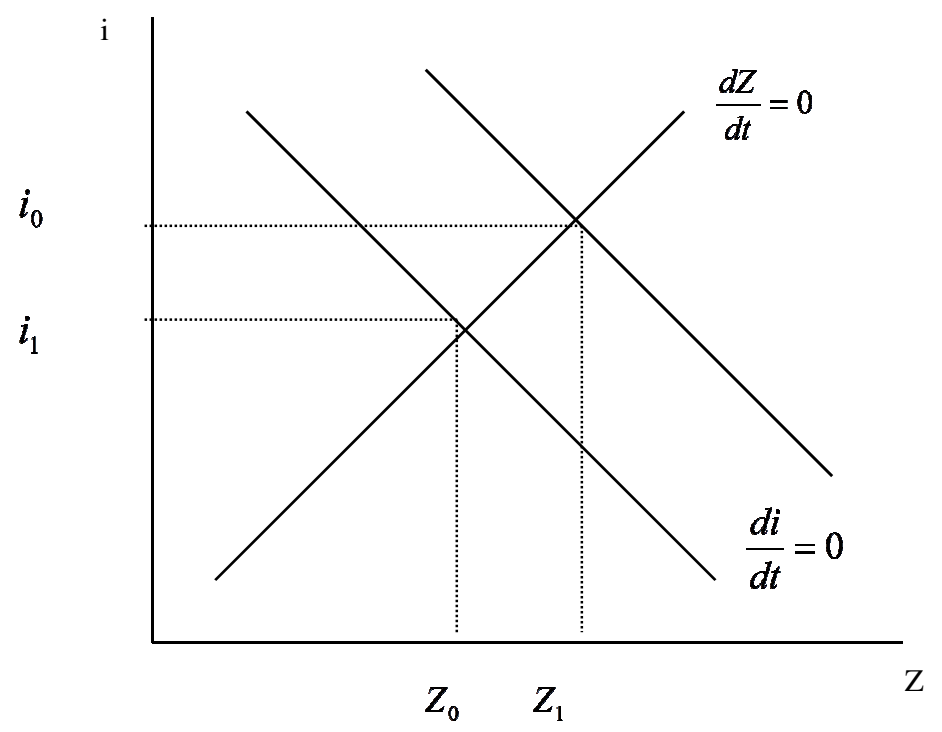

\section{Conclusão}

Ao longo do presente artigo foi desenvolvido um modelo dinâmico não linear de inspiração pós-keynesiana para avaliar a performance, no curto e no longo prazo, de uma série de variáveis macroeconômicas em um contexto de mobilidade (limitada) de capitais. O modelo aqui apresentado mostra que, no curto prazo, um aumento do endividamento externo tem impacto positivo sobre a performance macroeconômica. Mais especificamente, um aumento do endividamento externo gera um aumento do valor de equilíbrio de curto prazo do grau de utilização da capacidade produtiva e da taxa de crescimento do estoque de capital. Contudo, no longo prazo, a abertura da conta de capitais do balanço de pagamentos pode ter efeitos desestabilizadores sobre o sistema econômico. Além disso, a análise de longo 
prazo do sistema econômico mostrou que uma redução da meta de inflação a ser perseguida pela autoridade monetária produz uma elevação da taxa real de juros e do endividamento externo de equilíbrio de longo prazo. Dessa forma, uma política monetária contracionista estará associada a um aumento permanente do custo de oportunidade do capital (podendo ter um efeito negativo sobre o crescimento de longo prazo) e um aumento da fragilidade externa da economia.

Ademais, analisou-se a consistência das estratégias de crescimento das economias desenvolvidas e em desenvolvimento. No geral, os resultados encontrados mostram a validade da Lei de Thirlwall, de modo que o regime de crescimento das economias depende da elasticidade-renda da demanda de importação e exportação. Para economias abertas desenvolvidas, o modelo de crescimento wage-led é perfeitamente plausível, porque uma expansão dos salários na renda é acompanhada de aumento do grau de utilização da capacidade produtiva e do crescimento, observando-se o tradicional trade-off entre crescimento e inflação. Já para os países em desenvolvimento, a restrição a essa estratégia reside nos problemas estruturais de balanço de pagamentos. Nesse sentido, argumenta-se que uma estratégia consistente para esses países, compatível com as restrições externas estruturais relacionadas ao balanço de pagamentos, é o regime do tipo profit-led, dado a relação inversa entre crescimento dos salários e câmbio real.

\section{Referências bibliográficas}

BERTELLA, M. A; LIMA, G. T. Termos de troca, salário real e nível de atividade. Revista da Sociedade Brasileira de Economia Política, São Paulo, n. 9, p. 86-106, 2001.

BHADURI, A.; MARGLIN, S. Unemployment and the real wage: the economic basis for contesting political ideologies. Cambridge Journal of Economics, v. 14, n. 4, p. 375-393, 1990.

BHAGWATI, J. Yes to free trade, maybe to capital controls. Wall Street Journal, A38, Nov. 16, 1998.

BLANCHARD, O Macroeconomia. Rio de Janeiro: Campus, 2001.

BLECKER, R. A. International competition, income distribution and economic growth. Cambridge Journal of Economics, v. 13, p. 395-412, 1989.

The balance-of-payments-constrained growth model and the limits to export-led growth. In: DAVIDSON, P. (Ed.). A post Keynesian perspective on twenty-first century economic problems. Cheltenham, UK and Northampton, US: Edward Elgar, 2002. p. 69-88. 
BORTIS, H. Institutions, behaviour and Economic Theory - A contribution to classical-Keynesian political economy. Cambridge: Cambridge University Press, 1997.

BRESSER, L. C.; NAKANO, Y. Crescimento econômico com poupança externa? Revista de Economia Política, v. 23, n. 2, 2003.

CALVO, G. Crises de balanços de pagamentos em mercados emergentes. In: KRUGMAN, P. (Org.). Crises monetárias. São Paulo: Makron Books, 2001.

CARVALHO, L.; REZAI, A. Personal income inequality and aggregate demand. Paper presented at the annual conference of the Research Network in macroeconomics and Macroeconomic Policies, Berlin, Germany, October 24-25, 2013.

DUTT, A. K. Growth, distribution and uneven development. Cambridge: Cambridge University Press, 1990.

FLEMING, J. M. Domestic financial policies under fixed and under flexible exchange rates. International Monetary Fund Staff Papers, 9, 1962.

FOLEY, D. K.; MICHL, T. R. Growth and distribution. Cambridge, MA: Harvard University Press, 1999.

KALDOR, N. Alternative theories of distribution. Review of Economic Studies, v. 23, n. 2, 1956.

A model of economic growth. Economic Journal, 67, 1957.

KALECKI, M. The theory of economic dynamics. London: Allen \& Unwin, 1954. . Selected essays on the dynamics of the capitalist economy. Cambridge: Cambridge University Press, 1971.

LOPES, J. Crises econômicas na América Latina: algumas considerações à luz da teoria de M. Kalecki. São Paulo: Edusp, 2001.

LUCAS JR., R. E. Why doesn't capital flow from rich to poor countries? American Economic Review Paper and Proceedings, 90, p. 92-96, 1990.

MARGLIN, S. Growth, distribution, and prices. Cambridge, MA: Harvard University Press, 1984.

MARQUETTI, A. A. Do rising real wages increase the rate of labor-saving technical change? Some econometric evidence. Metroeconomica, v. 55, n. 4, p. 432-441, 2004.

MARX, K. O Capital. Rio de Janeiro: Civilização Brasileira, 1988. 
MCKINNON, R. Money and capital in economic development. Washington, DC: Brookings Institution, 1973.

. The order of economic liberalization: financial control in the transition to a market economy. Baltimore: John Hopkins University Press, 1991.

MUNDELL, R. The appropriate use of monetary and fiscal policy for internal and external stability. International Monetary Fund Staff Papers, 9, 1962.

OREIRO, J. L. Poupança externa e performance macroeconômica: uma análise a partir de um modelo macrodinâmico não-linear de acumulação de capital e endividamento externo. Revista de Economia Política, v. 24, n. 2, 2004.

PALLEY, T. I. The middle class in macroeconomics and growth theory: a three class neo-Kaleckian-Goodwin model. Cambridge Journal of Economics, web access, Jul., 2014.

PASINETTI, L. The rate of profit and income distribution in relation to the rate of economic growth. Review of Economic Studies, 29, 1962.

ROBINSON, J. The accumulation of capital. London: Macmillan, 1956.

A model of accumulation. In: SEN, A. (Org.). Growth economics. Middlesex: Penguim Books, 1962. [Ano da edição: 1970].

RODRIK, D. Who needs capital-account convertibility? In: KENEN, P. Should the IMF pursue capital-account convertibility? Princeton, NJ: Princeton University. International Finance Section, Department of Economics, 1998. (Essays in International Finance, n. 207).

ROWTHORN, R. Demand, real wages and economic growth. Thames Papers in Political Economy, Autumn, p. 1-39, 1981, reprinted in Studi Economici, v. 18, p. 3-54, 1982.

Unemployment, wage bargaining and capital-labour substitution. Cambridge Journal of Economics, v. 23, p. 413-425, 1999.

SHAW, E. Financial deepening in economic development. New York: Oxford University Press, 1973.

SIMONSEN, M. H; CYSNE, R. P. Macroeconomia. São Paulo: Ed. Atlas, 1995.

SOLOW, R. A contribution to the theory of economic growth. Quarterly Journal of Economics, v. 70, 1956.

STEINDL, J. Maturity and stagnation in American capitalism. New York: Monthly Review Press, 1952.

TAKAYAMA, A. Analytical methods in economics. Michigan: The University of Michigan Press, 1993. 
Flávio A. C. Basilio / José Luís da Costa Oreiro

TAYLOR, L. A stagnationist model of economic growth. Cambridge Journal of Economics, v. 9, p. 383-403, 1985.

. Inflation, income distribution, and growth. Cambridge, MA: MIT Press,

1991.

. Varieties of stabilization experience. Oxford: Clarendon Press, 1998. 